Check for updates

Cite this: RSC Adv., 2017, 7, 43621

Received 27th March 2017 Accepted 28th August 2017

DOI: $10.1039 / \mathrm{c} 7 \mathrm{ra03521g}$

rsc.li/rsc-advances

\section{Comparative intestinal bacteria-associated pharmacokinetics of 16 components of Shengjiang Xiexin decoction between normal rats and rats with irinotecan hydrochloride (CPT-11)-induced gastrointestinal toxicity in vitro using salting-out sample preparation and LC-MS/MS $\uparrow$}

\author{
Huanyu Guan, (D) ab Xiaoming Wang, ${ }^{a}$ Shiping Wang, ${ }^{\mathrm{b}}$ Yang He, ${ }^{\mathrm{a}}$ Jiajing Yue, ${ }^{\mathrm{a}}$ \\ Shanggao Liao, ${ }^{b}$ Yuanda Huang ${ }^{a}$ and Yue Shi ${ }^{\star a}$
}

\begin{abstract}
Shengjiang Xiexin decoction (SXD) exerts protective effects against gastrointestinal injury induced by irinotecan hydrochloride (CPT-11). The intestinal bacteria-associated in vitro pharmacokinetics of 16 components of SXD in normal rats and those with CPT-11-induced gastrointestinal toxicity were compared in this study. A sensitive and reproducible ultra-high-performance liquid chromatography coupled to tandem mass spectrometry (UHPLC-MS/MS) method was developed for the quantification of 16 components of SXD in a rat intestinal bacteria incubation system, using naringin, naringenin and tetrahydropalmatine as internal standards (ISS). The samples were prepared via salting-out assisted liquid-liquid extraction (SALLE) with $\mathrm{NaCl}$ to reduce matrix effects. Chromatographic separation was performed on a sub- $2 \mu \mathrm{m}$ analytical column with acetonitrile and $0.1 \%$ aqueous formic acid as mobile phase. All of the analyzed components and ISs were detected via multiple reaction monitoring (MRM) scanning with electrospray ionization. The proposed method was successfully applied for the in vitro pharmacokinetic analysis of the multiple components of a complex mixture consisting of a traditional Chinese medicine (TCM) and an intestinal bacterial incubation system. The pharmacokinetic parameters of some flavonoid glycosides and aglycones in the rats with CPT-11-induced gastrointestinal toxicity were significantly different $(p<0.05, p<0.01$ ) from those in the normal rats, which suggested that consumption of CPT-11 could qualitatively and/or quantitatively alter the intestinal bacteria as well as the metabolic activities of enzymes. The in vitro pharmacokinetic analysis of these components in the intestinal bacterial incubation system provided valuable information for achieving a deeper understanding of the mechanisms involved in the alteration of intestinal bacteria induced by CPT-11 and further in vivo pharmacokinetic research on SXD. The intestinal bacteria-based pharmacokinetic method could benefit the study of interactions between TCMs and chemical drugs in clinical use.
\end{abstract}

\section{Introduction}

Irinotecan hydrochloride (CPT-11) is a promising antitumor derivative of camptothecin, a topoisomerase I inhibitor. ${ }^{1}$ However, at higher dosages, CPT-11 can cause severe and uncontrollable diarrhea, which is one of the main side-effects of CPT-11 and has impeded its utilization in more aggressive

${ }^{a}$ Institute of Medicinal Plant Development, Chinese Academy of Medical Sciences and Peking Union Medical College, Beijing 100193, China.E-mail: shiyue1029@126.com; Fax: +86-10-57833270; Tel: $+86-10-57833255$

${ }^{b}$ School of Pharmaceutical Sciences, Guizhou Medical University, Guiyang 550004, China

$\dagger$ Electronic supplementary information (ESI) available. See DOI: $10.1039 / \mathrm{c} 7 \mathrm{ra03521g}$ antitumor regimens. ${ }^{2,3} \mathrm{CPT}-11$ is converted by carboxylesterase enzymes mainly in the liver into its active metabolite, $\mathrm{SN}-38$, which is considered to be responsible for the induction of diarrhea. ${ }^{4} \mathrm{SN}-38$ is then detoxified to $\mathrm{SN}-38$ glucuronide (SN-38G) by UDP-glucuronosyltransferase, and is excreted via bile. ${ }^{5} \mathrm{SN}-38 \mathrm{G}$ excreted into the intestinal lumen through bile may be deconjugated by bacterial $\beta$-glucuronidase, releasing $\mathrm{SN}-38$, which leads to the accumulation of $\mathrm{SN}-38$ in the intestine. ${ }^{6} \mathrm{SN}-38$ in the cecal and colonic contents directly damages the intestinal epithelium and induces delayed-onset diarrhea. ${ }^{7}$ Bacterial $\beta$-glucuronidase is involved in the metabolism of CPT11 and plays a crucial role in the intestinal toxicity of CPT- $11 .^{8}$ Many microflora in the gastrointestinal tract can produce $\beta$-glucuronidase. Changes in the intestinal microflora occur 
after CPT-11 treatment, and an increase in the expression of $\beta$ glucuronidase has been observed.9,10 Moreover, the translocation of special bacteria induced by CPT-11 can cause infection. ${ }^{11}$ Therefore, antibiotics have been used to reduce the level of the microflora and decrease the bacterial $\beta$-glucuronidase activity in the gastrointestinal tract to alleviate the CPT-11induced diarrhea. ${ }^{\mathbf{2 , 6 , 1 2}}$ Nevertheless, there are some obvious drawbacks to the antidiarrheal treatment, which is detrimental to commensal bacteria. Admittedly, intestinal bacteria are involved in the metabolism of carbohydrate, the production of vitamins, and the processing of bile acids, sterols, and xenobiotics. Fortunately, potent bacterial $\beta$-glucuronidase inhibitors that do not affect commensal bacteria have been identified. ${ }^{13}$

Traditional Chinese Medicine (TCM) has been used to treat or prevent cancer-related symptoms and chemotherapy-associated toxicity for thousands of years. Most of TCM are orally administered in the form of decoctions and are therefore inevitably brought into contact with bacteria and enzymes in the alimentary tract. As described in "Shang Han Lun", Shengjiang Xiexin decoction (SXD) is a classic TCM formula to be used for the treatment of gastroenteritis, ulcerative colitis and diarrhea, ${ }^{\mathbf{1 4}}$ consisting of eight herbs in the ratio of $9: 9: 3: 12: 3: 9: 9: 12$ on a dry weight basis: Pinellia ternata ("banxia" in Chinese, the rhizome of $P$. ternata (Thunb.) Breit.), Glycyrrhiza uralensis ("gancao" in Chinese, the radix of G. uralensis Fisch.), Coptis chinensis ("huanglian" in Chinese, the rhizome of $C$. chinensis Franch.), Ziziphus jujuba ("dazao" in Chinese, the fruit of Z. jujuba Mill.), Zingiber officinale ("ganjiang" in Chinese, the rhizome of $Z$. officinale Rosc.), Scutellaria baicalensis ("huangqin” in Chinese, the radix of $S$. baicalensis Georgi.), Codonopsis pilosula ("dangshen" in Chinese, the radix of C. pilosula (Franch.) Nannf.) and Zingiberis recens ("shengjiang" in Chinese, the rhizome of $Z$. recens.). The combination of these herbs is based upon the rule of "Jun-Chen-Zuo-Shi", known as "Emperor-Minister-AssistantCourier". Among them, C. chinensis and S. baicalensis serve as "Jun" and "Chen" to alleviate the gastrointestinal toxicity, respectively. ${ }^{15}$<smiles>O=C(O)[C@H]1O[C@H](Oc2cc3oc(-c4ccccc4)cc(=O)c3c(O)c2O)[C@H](Oc2cc3oc(-c4ccc(O)cc4)c(O)c(O)c-3c(=O)c2)[C@H](O)[C@@H](O)[C@@H]1O</smiles><smiles>COc1c(O[C@@H]2OC(C(=O)O)[C@@H](O)[C@H](O)[C@H]2O)cc(O)c2c(=O)cc(-c3ccccc3)oc12</smiles><smiles>COc1c(O)cc2oc(-c3ccccc3)cc(=O)c2c1O</smiles><smiles>COc1c(O)cc(O)c2c(=O)cc(-c3ccccc3)oc12</smiles><smiles>O=c1cc(-c2ccccc2)oc2cc(O)cc(O)c12</smiles><smiles>O=c1cc(-c2ccccc2)oc2cc(O)c(O)c(O)c12</smiles><smiles>O=CC=Cc1ccc(O[C@@H]2OC(CO)[C@H](O)C(O)C2O)cc1</smiles>
wogonin chrysin baicalein<smiles>O=C(/C=C/c1ccc(O)cc1)c1ccc(O)cc1O</smiles><smiles>O=C1C[C@H](c2ccc(OC3OC(CO)C(O)C(O)C3O)cc2)Oc2cc(O)ccc21</smiles>
isoliquiritin isoliquiritigenin

liquiritin<smiles>O=C1C[C@@H](c2ccc(O)cc2)Oc2cc(O)ccc21</smiles><smiles></smiles><smiles>COCOc1cc2c(cc1OC)-c1cc3ccc4c(c3c[n+]1CC2)OCO4</smiles><smiles>COc1cc2c(cc1O)CC[n+]1cc3c(OC)c(OC)ccc3cc1-2</smiles>
berberine<smiles>[13CH3][13CH3]</smiles><smiles>C[C@@H](O)[C@H](O)[InH2]</smiles>
jatrorrhizine<smiles>COc1cc2c(cc1OC)-c1cc3ccc(OC)c(OC)c3c[n+]1CC2</smiles>

Fig. 1 The chemical structures of 16 components. 
Regarding modern clinical practice, when patients in several hospitals were orally administered SXD two days prior to the initiation of chemotherapy to prevent CPT-11-induced gastrointestinal toxicity, it was found that this treatment reduced the incidence of diarrhea. ${ }^{16}$ Moreover, SXD has been reported to regulate the CPT-11-induced apoptosis and necrosis of intestinal mucosal and functional cells. ${ }^{17}$ It is also noteworthy that SXD can decreased the activity of $\beta$-glucuronidase after irinotecan administration. ${ }^{18}$ Baicalin, a known flavonoid in SXD, is a $\beta$-glucuronidase inhibitor ${ }^{19}$ that inhibits the uptake of SN-38 in a concentration-dependent manner. ${ }^{20}$ Moreover, as a traditional medicines, SXD is composed of multiple components, and the flavonoids, alkaloids and triterpenoid saponins in SXD are considered the most important active components of the mixture. ${ }^{21-24}$ Some flavonoids, alkaloids and saponins can be transformed by intestinal bacteria ${ }^{25-28}$ to their metabolites, which exhibit different pharmacological activities. However, the intestinal bacteria-associated pharmacokinetics of these components in vitro are not clear. In addition, the co-existence of multiple compounds in TCMs and chemical drugs may lead to the intestinal bacteria-based metabolic and pharmacokinetic interactions. There are few available studies on such interactions involving intestinal bacteria because of the complexity of the chemical components of TCM and the intestinal bacteria system.

In a previous study, we carried out the simultaneous quantification of 14 constituents of SXD using UFLC-MS/MS. ${ }^{29}$ An analytical method has also been developed for the simultaneous quantification of flavonoids, alkaloids and triterpenoid saponins in Banxia xiexin decoction, which is analogous to SXD formula. ${ }^{30}$ Analytical conditions for the individual determination of several flavonoids, ${ }^{21,28,31,32}$ alkaloids, ${ }^{33,34}$ and triterpenoid saponins $^{35,36}$ in biological matrices have been reported. However, there is little available information about the quantification and in vitro pharmacokinetics of flavonoids, alkaloids and triterpenoid saponins from TCM formulas in complex intestinal bacterial incubation systems. In addition, no data on the intestinal bacteria-associated pharmacokinetics of the major components of SXD under interaction with CPT-11 have been reported.

In the present study, a sensitive, specific and precise method was established for the simultaneous determination of oroxylin A, baicalin, baicalein, wogonoside, wogonin, chrysin, scutellarin, isoliquiritin, isoliquiritigenin, berberine, coptisine, palmatine, jatrorrhizine, glycyrrhizic acid, liquiritin and liquiritigenin (Fig. 1) in an in vitro rat intestinal bacterial incubation system, via one sample preparation combined with two chromatographic conditions. The method was validated and utilized to compare the intestinal bacteria-associated pharmacokinetics of 16 components of SXD in vitro between normal rats and those with CPT-11-induced gastrointestinal toxicity.

\section{Experimental}

\subsection{Materials and reagents}

All medicinal plants were purchased from Huamiao Traditional Chinese Medicine Engineering Technology Development
Center (Beijing, China). The reference standards of baicalin, glycyrrhizic acid, baicalein, liquiritin, berberine, palmatine, naringin (used as an internal standard, IS1), naringenin (IS2) and tetrahydropalmatine (IS3) were purchased from the National Institute for the Control of Pharmaceutical and Biological Products (Beijing, China). Oroxylin A, wogonin, jatrorrhizine, scutellarin, wogonoside, isoliquiritin, isoliquiritigenin, chrysin and liquiritigenin (purity $\geq 98 \%$ ) were purchased from Shanghai Yuanye Biological Technology Co. Ltd. (Shanghai, China). Coptisine (purity $\geq 98 \%$ ) was purchased from Aladdin Industrial Co., Ltd. (Shanghai, China), and Campo® (CPT-11) for injection was obtained from Pfizer (Bentley, WA, Australia). General anaerobic medium broth (GAM broth) was purchased from Shanghai Kayon Biological Technology Co., Ltd. (Shanghai, China). Anaerobic packs were purchased from Mitsubishi Gas Chemical Company (Tokyo, Japan).

HPLC-grade methanol and acetonitrile were obtained from Honeywell Burdick \& Jackson Company (Morristown, NJ, USA). Formic acid (MS grade) was purchased from Fisher Scientific (Madrid, Spain). Deionized water for HPLC analysis was prepared using a Milli-Q water purification system (Millipore, Milford, MA, USA). All other reagents were of analytical grade.

\subsection{Equipment and LC-MS/MS conditions}

For LC-MS/MS, a Shimadzu LC-30AD series UHPLC (Shimadzu, Kyoto, Japan) consisting of a dual solvent delivery system (two LC-30AD pumps), refrigerated auto-sampler (SIL-30AC), column oven (CTO-20AC) and degasser (DGU-20A5R) was employed, which was coupled to an AB SCIEX Qtrap 4500 system (AB SCIEX, Foster City, CA, USA) equipped with an electrospray ionization source (Turbo Ionspray) for mass spectrometric detection. Data analysis was performed using AB SCIEX Analyst 1.6 Software (AB SCIEX).

The UHPLC separation was achieved on an ACQUITY UPLC ${ }^{8}$ BEH C18 column $(2.1 \mathrm{~mm} \times 100 \mathrm{~mm}, 1.7 \mu \mathrm{m})$ using acetonitrile (A) and $0.1 \%$ aqueous formic acid (B) as the mobile phase at a flow rate of $0.3 \mathrm{~mL} \mathrm{~min}{ }^{-1}$. The injection volume was set to $10 \mu \mathrm{L}$. The auto-sampler was conditioned at $10{ }^{\circ} \mathrm{C}$. All analyzed components were quantified in multiple reaction monitoring (MRM) mode. The optimized conditions were as follows: curtain gas (CUR): 10.0 psi; collision gas (CAD): medium; IonSpray voltage (IS): $-4500 \mathrm{~V}$ (in negative ionization mode) and $4500 \mathrm{~V}$ (in positive ionization mode); source temperature: $500{ }^{\circ} \mathrm{C}$; GS1: $40 \mathrm{psi}$; and GS2: 40 psi. The MS/MS transitions $(\mathrm{m} / \mathrm{z})$, declustering potentials (DP), collision energies (CE), entrance potentials (EP) and collision cell exit potentials (CXP) of the analyzed components and ISs are listed in Table 1. Two gradient elution programs were employed for different compounds in different ion modes. Oroxylin A, baicalin, baicalein, wogonoside, wogonin, chrysin, scutellarin, glycyrrhizic acid, liquiritin, liquiritigenin, naringin (IS1) and naringenin (IS2) were detected in negative ionization mode with elution program I: $5-5 \% \mathrm{~A}$ at $0-1 \mathrm{~min} ; 5-15 \% \mathrm{~A}$ at $1-3 \mathrm{~min} ; 15-15 \% \mathrm{~A}$ at 3-5 min; $15-20 \%$ A at $5-8 \mathrm{~min} ; 20-20 \%$ A at $8-11 \mathrm{~min} ; 20-35 \% \mathrm{~A}$ at $11-15 \mathrm{~min} ; 35-45 \% \mathrm{~A}$ at $15-20 \mathrm{~min} ; 45-100 \% \mathrm{~A}$ at $20-23 \mathrm{~min}$; $100-100 \% \mathrm{~A}$ at $23-26 \mathrm{~min} ; 100-5 \% \mathrm{~A}$ at $26-26.1 \mathrm{~min}$ and $5-5 \% \mathrm{~A}$ 
Table 1 MRM parameters of 16 compounds and three internal standards (ISs)

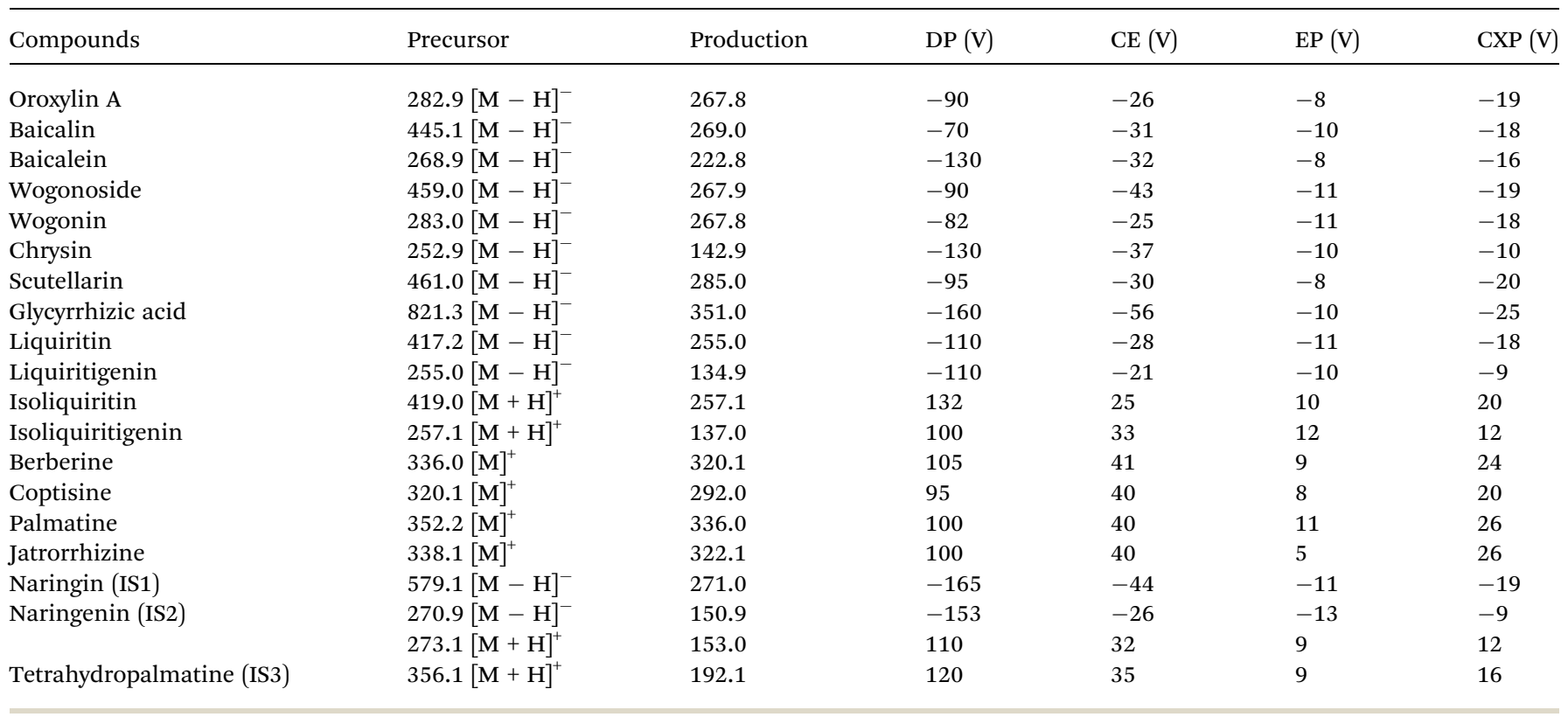

at 26.1-28 $\mathrm{min}$, while isoliquiritin, isoliquiritigenin, berberine, coptisine, palmatine, jatrorrhizine, naringenin (IS2) and tetrahydropalmatine (IS3) were detected in the positive ionization mode with elution program II: $5-5 \% \mathrm{~A}$ at $0-1 \mathrm{~min} ; 5-15 \% \mathrm{~A}$ at 1-3 $\mathrm{min} ; 15-15 \%$ A at 3-5 $\mathrm{min} ; 15-20 \%$ A at 5-9 $\mathrm{min} ; 20-20 \% \mathrm{~A}$ at $9-12 \mathrm{~min} ; 20-25 \% \mathrm{~A}$ at $12-16 \mathrm{~min} ; 25-45 \% \mathrm{~A}$ at $16-21 \mathrm{~min}$; $45-100 \%$ A at $21-23 \mathrm{~min} ; 100-100 \% \mathrm{~A}$ at $23-26 \mathrm{~min} ; 100-5 \% \mathrm{~A}$ at $26-26.1 \mathrm{~min}$ and $5-5 \% \mathrm{~A}$ at $26.1-28 \mathrm{~min}$.

\subsection{Preparation of standards and quality control samples}

Stock solutions of the standards were prepared by individually dissolving 16 reference substances in methanol to obtain a final concentration of $1.0 \mathrm{mg} \mathrm{mL}^{-1}$. These stock solutions were mixed to obtain a final mixed stock solution by adding the appropriate volumes. The mixed stock solution was serially diluted with $50 \%$ methanol to obtain working standard solutions with the desired concentrations. The required IS stock solutions containing tetrahydropalmatine $\left(0.25 \mu \mathrm{g} \mathrm{mL}^{-1}\right)$, naringin $\left(1.0 \mu \mathrm{g} \mathrm{mL} \mathrm{m}^{-1}\right)$ and naringenin $\left(1.0 \mu \mathrm{g} \mathrm{mL} \mathrm{m}^{-1}\right)$ were prepared in $50 \%$ methanol and used at concentrations of 25,100 and $100 \mathrm{ng} \mathrm{mL}^{-1}$, respectively, in each working solution and sample.

Quality control (QC) samples for each compound were prepared by spiking $100 \mu \mathrm{L}$ of the standard working solutions into $1 \mathrm{~mL}$ of the blank incubation solution for intestinal bacteria inactivated by acetonitrile/water-saturated $n$-butanol ( $1: 1, \mathrm{v} / \mathrm{v}$ ), to obtain the following concentrations (LLOQ, low, medium and high concentrations): $10,20,100$ and $400 \mathrm{ng} \mathrm{mL}$ for oroxylin A; 20, 40, 200 and $800 \mathrm{ng} \mathrm{mL} \mathrm{m}^{-1}$ for baicalin, baicalein, wogonoside and wogonin; 2, 4, 40, and $160 \mathrm{ng} \mathrm{mL}^{-1}$ for chrysin and jatrorrhizine; 3, 6, 60 and $240 \mathrm{ng} \mathrm{mL}^{-1}$ for scutellarin; 12, 24, 120 and $480 \mathrm{ng} \mathrm{mL}^{-1}$ for glycyrrhizic acid; 8, 16, 160 and $640 \mathrm{ng} \mathrm{mL} \mathrm{m}^{-1}$ for liquiritin; 10, 20, 200 and $800 \mathrm{ng} \mathrm{mL}$ for liquiritigenin; 3, 6, 60 and $240 \mathrm{ng} \mathrm{mL}^{-1}$ for isoliquiritin; 25, 50,200 and $400 \mathrm{ng} \mathrm{mL}^{-1}$ for coptisine and palmatine; 50, 100,
400 and $800 \mathrm{ng} \mathrm{mL} L^{-1}$ for berberine and 1, 2, 20 and $80 \mathrm{ng} \mathrm{mL} \mathrm{m}^{-1}$ for isoliquiritigenin.

\subsection{Preparation of SXD}

The mixture of the eight crude herbal drugs was prepared according to the previously described formula, which was immersed in distilled water for $30 \mathrm{~min}$. Next, a ten-fold volume of water was added, and the resulting mixture was decocted twice by boiling for $1 \mathrm{~h}$. After filtration, the filtrates were combined, evaporated, and concentrated to form an extract. The obtained SXD extract $(1.0 \mathrm{~g})$ was extracted with $100 \mathrm{~mL}$ of $60 \%$ methanol and was ultrasonicated for $30 \mathrm{~min}$. After centrifugation at $14000 \mathrm{rpm}$ for $10 \mathrm{~min}, 10 \mu \mathrm{L}$ of the supernatant was injected into the LC-MS/MS system to analyze the content of all 16 constituents in the SXD powder. The contents of oroxylin A, baicalin, baicalein, wogonoside, wogonin, chrysin, scutellarin, glycyrrhizic acid, liquiritin, liquiritigenin, isoliquiritin, isoliquiritigenin, berberine, coptisine, palmatine and jatrorrhizine, were determined to be $202.74 \pm 28.41$, $17990.87 \pm 1678.98,215.63 \pm 25.20,13744.29 \pm 1872.70$, $242.92 \pm 15.50,12.56 \pm 1.36,578.08 \pm 76.20,2132.42 \pm 316.42$, $1821.92 \pm 148.52,11.32 \pm 0.65,564.84 \pm 38.10,20.05 \pm 2.13$, $7981.74 \pm 981.55,4036.53 \pm 361.63,3073.06 \pm 471.40$, and $813.70 \pm 78.78 \mathrm{mg} \mathrm{kg}^{-1}$, respectively.

\subsection{Animal handling}

Thirty male Sprague-Dawley rats (weighing $200 \pm 20 \mathrm{~g}$ ), which were purchased from Department of Laboratory Animal Science of Peking University Health Center (Beijing, China), were maintained under a standard 12/12 h-light/dark cycle, at 20-25 ${ }^{\circ} \mathrm{C}$ and $40-60 \%$ humidity, with water and food available ad libitum for one week to adapt to the environment prior to the experiment. All experiments were performed according to the National Institutes of Health Guidelines for Animal Research 
and were approved by the Ethics Committee of the Institute of Medicinal Plant Development, CAMS \& PUMC.

The animals were randomly divided into group GT and C $(n=15)$. CPT-11 was administered intravenously (i.v.) at a dose of $60 \mathrm{mg}$ per $\mathrm{kg}$ per day to the group GT rats via the tail vein for four consecutive days, ${ }^{3}$ while corresponding administration of saline was performed in group $\mathrm{C}$ rats. Body weight and diarrhea scores $^{3}$ were monitored throughout the experimental period. Briefly, the severity of diarrhea was scored as follows: 0 , normal; 1 , soft feces or small black feces; 2 , muddy feces; 3 , watery feces or mucous feces. Animals were sacrificed by cervical dislocation under anesthesia. The colonic contents were collected aseptically in a sterile container $72 \mathrm{~h}$ after the final administration.

\subsection{Incubation experiments}

Samples of $2.0 \mathrm{~g}$ of the colonic contents were immediately mixed initially with $8 \mathrm{~mL}$ of aseptic physiological saline, and homogenized using a vortex-mixer. The homogenized mixture was centrifuged at $2000 \mathrm{rpm}$ for $10 \mathrm{~min}$, and $5 \mathrm{~mL}$ of the suspension was inoculated into 45 volumes of GAM broth, which was then incubated at $37{ }^{\circ} \mathrm{C}$ in an anaerobic pack for $24 \mathrm{~h}$. The resulting mixture of bacteria was centrifuged at $4000 \mathrm{rpm}$ for $10 \mathrm{~min}$, and the residue was suspended in $5 \mathrm{~mL}$ of aseptic physiological saline to be used as the intestinal bacterial mixture.

The intestinal bacterial mixture was inoculated into GAM broth in the presence of SXD extract $\left(351.6 \mu \mathrm{g} \mathrm{mL} L^{-1}\right)$ in a ratio of $1: 4(\mathrm{v} / \mathrm{v})$. The cultured mixture was incubated anaerobically at $37^{\circ} \mathrm{C}$. Finally, a $1 \mathrm{~mL}$ aliquot of the cultured mixture was taken out, and the reaction was terminated by adding an equivalent volume of acetonitrile/water-saturated $n$-butanol $(1: 1, \mathrm{v} / \mathrm{v})$ at 0 , $2,4,6,8,12,16,20,24,28,32,36$ and $48 \mathrm{~h}$.

\subsection{Sample preparation}

Salting-out assisted liquid-liquid extraction (SALLE) was employed for sample preparation. Phase separation in the terminated incubation mixture was induced by the addition of $\mathrm{NaCl}$ until saturation. The organic phase was transferred to a clean tube and was evaporated to dryness under a steady stream of nitrogen gas. The residue was reconstituted with $1 \mathrm{~mL}$ of $50 \%$ methanol, followed by centrifugation at $14000 \mathrm{rpm}$ for $10 \mathrm{~min}$. The supernatant was then transferred to an autosampler vial. First, $10 \mu \mathrm{L}$ of the supernatant was injected into the LC-MS/MS system for the simultaneous analysis of oroxylin A, baicalin, baicalein, wogonoside, wogonin, chrysin, scutellarin, glycyrrhizic acid, liquiritin, liquiritigenin, naringin (IS1) and naringenin (IS2) using elution program I. Next, another $10 \mu \mathrm{L}$ was injected into the LC-MS/MS system for the simultaneous analysis of isoliquiritin, isoliquiritigenin, berberine, coptisine, palmatine, jatrorrhizine, naringenin (IS2) and tetrahydropalmatine (IS3) under the chromatographic condition using elution program II.

\subsection{Method validation}

The method was validated in terms of selectivity, calibration curve, sensitivity, precision, accuracy, recovery, matrix effect, dilution integrity and stability, in accordance with the guideline for bioanalytical method validation (2011) from the European Medicines Agency (EMA). ${ }^{37}$

Selectivity. Three different internal standards were used during the method development process: naringin (IS1) for baicalin, wogonoside, scutellarin, glycyrrhizic acid, liquiritin and liquiritigenin; naringenin (IS2) for oroxylin A, baicalein, wogonin, chrysin, isoliquiritin and isoliquiritigenin; and tetrahydropalmatine (IS3) for berberine, coptisine, palmatine and jatrorrhizine. The selectivity of the method was evaluated by analyzing six individual batches of inactivated blank rat intestinal bacterial incubation solution. Each blank sample was tested for endogenous interferences using the proposed extraction procedure and LC-MS/MS conditions. The chromatogram of a blank sample was compared with that of the bacteria incubation solution spiked with the analyzed components and ISs and that of the samples obtained after incubation with intestinal bacteria for $2 \mathrm{~h}$.

Linearity and lower limit of quantification (LLOQ). The working standard solutions were spiked into blank intestinal bacterial incubation solution inactivated by acetonitrile/watersaturated $n$-butanol $(1: 1, \mathrm{v} / \mathrm{v})$ to prepare the calibration curves in the following ranges: $10-500 \mathrm{ng} \mathrm{mL}^{-1}$ for oroxylin $\mathrm{A}$; 20-1000 ng $\mathrm{mL}^{-1}$ for baicalin, baicalein, wogonoside and wogonin; 2-200 $\mathrm{ng} \mathrm{mL}^{-1}$ for chrysin and jatrorrhizine; 3-300 $\mathrm{ng} \mathrm{mL}^{-1}$ for scutellarin; $12-600 \mathrm{ng} \mathrm{mL}^{-1}$ for glycyrrhizic acid; 8-800 $\mathrm{ng} \mathrm{mL}^{-1}$ for liquiritin; $10-1000 \mathrm{ng} \mathrm{mL}^{-1}$ for liquiritigenin; 3-300 $\mathrm{ng} \mathrm{mL}^{-1}$ for isoliquiritin; $25-500 \mathrm{ng} \mathrm{mL}^{-1}$ for coptisine and palmatine; $50-1000 \mathrm{ng} \mathrm{mL^{-1 }}$ for berberine; and 1-100 $\mathrm{ng} \mathrm{mL} \mathrm{m}^{-1}$ for isoliquiritigenin.

For all 16 components, each calibration curve was constructed by plotting the peak area ratio of the analyzed component to the IS versus the nominal concentration of the analyzed component using a $1 / x$-weighted linear least-square regression model. The LLOQ was the lowest concentration of the analyzed component on the calibration curve with an acceptable accuracy and precision. The accuracy (relative error, $\mathrm{RE})$ of the LLOQ sample was within $\pm 20 \%$ and the precision (relative standard deviation, RSD) was less than $20 \%$.

Precision and accuracy. The intra-day and inter-day precision and accuracy were determined by quantifying four concentration levels of QC samples (five samples for each concentration level) on the same day and on three consecutive validation days, respectively. The intra-day and inter-day precision were expressed as the RSD and the accuracy was evaluated based on the RE. The RSD should not exceed 15\% and the RE values should be within $\pm 15 \%$, except for the RSD and RE of the LLOQ which should not exceed $20 \%$ and should be within $20 \%$, respectively.

The extraction recovery and matrix effect. The extraction recoveries of the analyzed components at three QC levels (low, medium and high concentrations) were evaluated by comparing the peak areas of the analyzed components of the extracted QC samples with those obtained from pure reference standards spiked into post-extracted blank samples at the equivalent concentration. 
Six batches of the blank intestinal bacterial incubation solution inactivated by acetonitrile/water-saturated $n$-butanol from individual rats were used to prepare QC samples at a low and a high level of concentration to evaluate the relative matrix effect. The matrix factor (MF) of each analyzed component for each batch was determined by calculating the ratio of the peak area of the analyzed component in the present of matrix to that in pure standard solutions. The IS-normalized MF was calculated by dividing the MF of the analyzed component by the MF of the IS. The RSD of the IS-normalized MF calculated from the six batches of the present matrix should not exceed $15 \%$.

$$
\text { IS normalised MF }=\frac{\text { MF of analyzed component }}{\text { MF of IS }}
$$

Dilution integrity. Five QC samples with concentrations that were two times greater than the upper limit of the calibration curve were prepared to evaluate the dilution integrity. These QC samples were diluted with post-extracted blank intestinal bacterial incubation solution containing the ISs at a dilution factor of ten to bring the concentration into the required calibration range. The precision (RSD) should not exceed $15 \%$, and the accuracy (RE value) should be within $15 \%$.

Stability. The stability of the analyzed components in inactivated blank intestinal bacterial incubation solution was evaluated by storing the QC samples at the three proposed concentrations (low, medium and high) under auto-sampler conditions $\left(10{ }^{\circ} \mathrm{C}\right)$ for $48 \mathrm{~h}$ (short-term stability). Long-term stability was determined by analyzing QC samples stored at $-80{ }^{\circ} \mathrm{C}$ for two months. Freeze and thaw stability was determined using QC samples after three freeze-thaw cycles $(-80$ to $20{ }^{\circ} \mathrm{C}$ ). These QC samples were analyzed against a calibration curve obtained from freshly prepared calibration standards, and the obtained concentrations were compared with those of freshly prepared QC samples. The observed deviations in the concentration were calculated as percentages to evaluate the stability.

\subsection{Data analysis}

All calibration and quantification data were calculated using $\mathrm{AB}$ SCIEX Analyst 1.6 Software. Pharmacokinetic parameters, area under the concentration time curve to the respective sampling point $\left(\mathrm{AUC}_{0-t}\right)$, mean resident time $\left(\mathrm{MRT}_{0-t}\right)$, half-life $\left(T_{1 / 2}\right)$ and clearance rate $(\mathrm{CL})$ for glycosides and alkaloids, and $\mathrm{AUC}_{0-t}$, maximum concentration $\left(C_{\max }\right)$ and time to achieve maximum concentration $\left(T_{\max }\right)$ for aglycones, were evaluated with Phoenix WinNonlin 6.0 (Certara, USA). An unpaired Student $t$-test was employed to compare the differences in pharmacokinetic parameters between group GT and C. Differences in body weight and diarrhea scores were analyzed via repeated measures analysis of variance and one-way ANOVA test.

\section{Results and discussion}

\subsection{Method development}

Sample preparation and analytical technology were optimized for the determination of target components in a complex

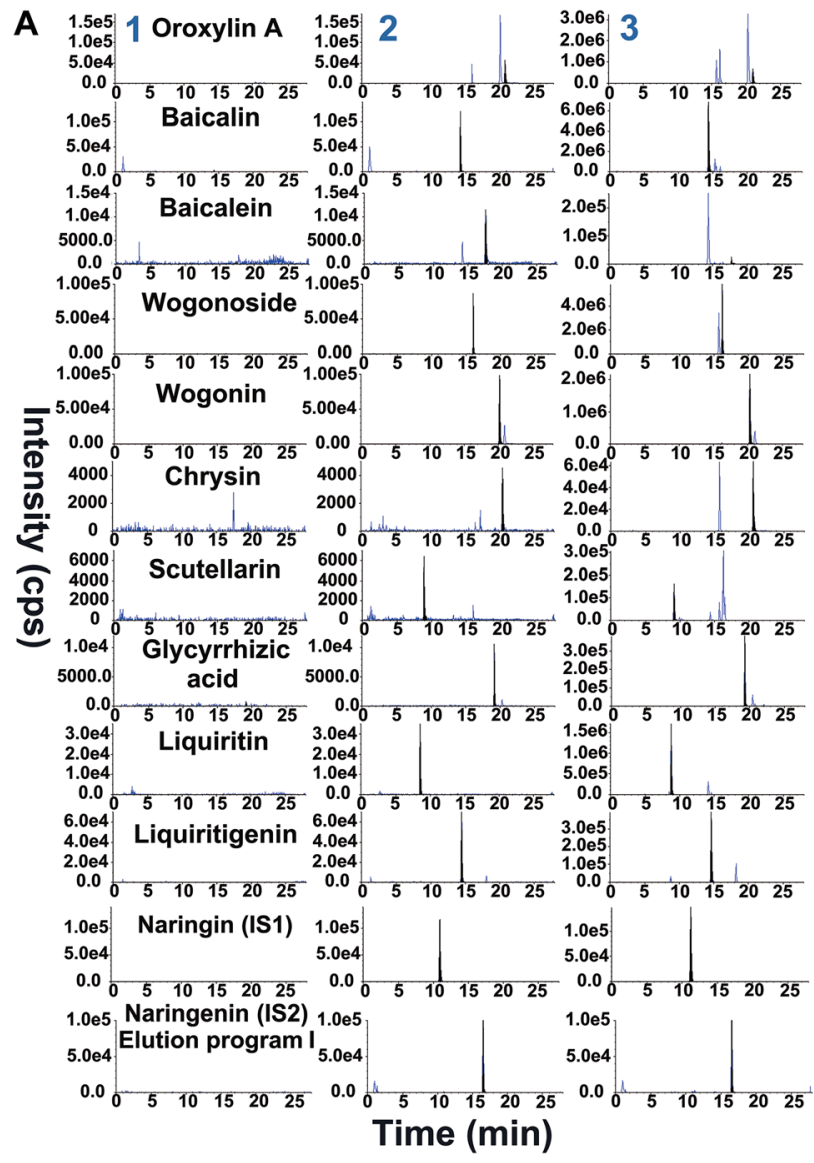

B

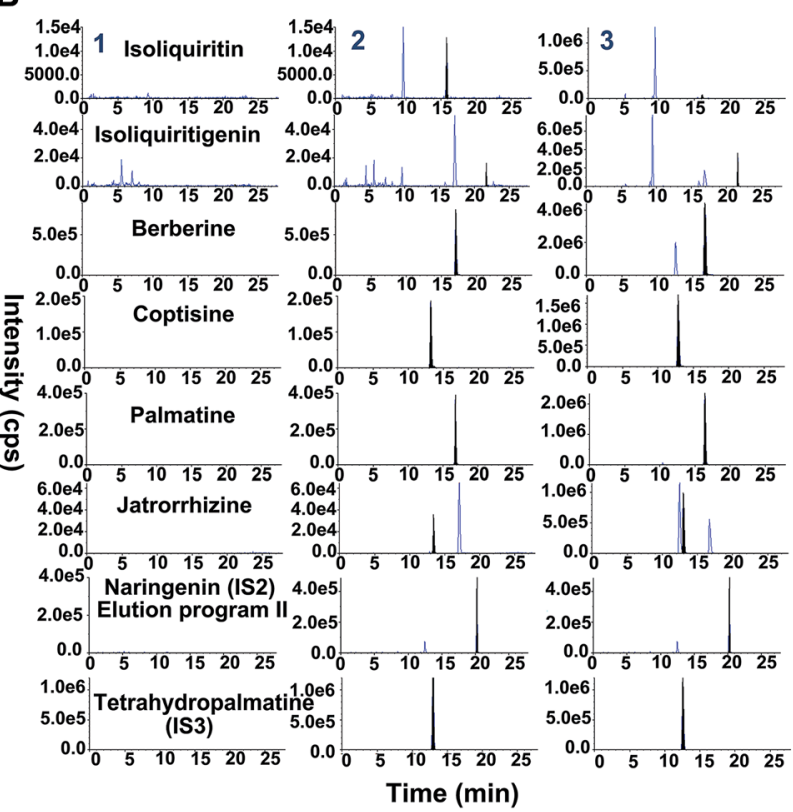

Fig. 2 (A) Representative MRM chromatograms of 10 components and two ISs in negative ion mode. (1) Blank incubation solution of bacteria; (2) blank incubation solution of inactivated bacteria spiked with the standard solutions and two ISs; (3) samples obtained after $2 \mathrm{~h}$ incubation in intestinal bacteria solution. (B) Representative MRM chromatograms of 6 components and two ISs in positive ion mode. (1) Blank incubation solution of bacteria; (2) blank incubation solution of inactivated bacteria spiked with the standard solutions and two ISs; (3) samples obtained after $2 \mathrm{~h}$ incubation in intestinal bacteria solution. 
Table 2 The calibration curves, linearity ranges and LLOQs of the 16 components

\begin{tabular}{llll}
\hline Components & Calibration equation & $r$ & Linear range $\left(\mathrm{ng} \mathrm{ml}^{-1}\right)$ \\
\hline Oroxylin A & $y=0.0518 x+0.558$ & 0.9994 & $10-500$ \\
Baicalin & $y=0.0125 x+0.071$ & 0.9987 & $20-1000$ \\
Baicalein & $y=0.00431 x+0.0765$ & 0.9989 & $20-1000$ \\
Wogonoside & $y=0.0129 x+0.0155$ & 0.9983 & $20-1000$ \\
Wogonin & $y=0.0195 x+0.167$ & 0.9988 & $20-1000$ \\
Chrysin & $y=0.0173 x+0.0127$ & 0.9993 & $2-200$ \\
Scutellarin & $y=0.00653 x+0.00312$ & 0.9990 & $3-300$ \\
Glycyrrhizic acid & $y=0.00378 x+0.006$ & 0.9992 & $12-600$ \\
Liquiritin & $y=0.00717 x+0.00829$ & 0.9994 & $8-800$ \\
Liquiritigenin & $y=0.0234 x+0.0888$ & 0.9993 & $10-1000$ \\
Isoliquiritin & $y=0.00153 x+0.00267$ & 0.9996 & $3-300$ \\
Isoliquiritigenin & $y=0.0249 x+0.00376$ & 0.9996 & $1-100$ \\
Berberine & $y=0.0115 x+0.127$ & 0.9994 & $50-1000$ \\
Coptisine & $y=0.00253 x+0.00777$ & 0.9993 & $25-500$ \\
Palmatine & $y=0.00623 x+0.0138$ & 0.9996 & $25-500$ \\
Jatrorrhizine & $y=0.00585 x+0.00156$ & 0.9988 & $2-200$
\end{tabular}

matrix. A TCM formula has been known as a complex system containing tens or even hundreds of different chemical constituents. The analysis of target components is interfered by the biological matrix and the other coexisting components in TCM formula. In the present study, it was important to take into account the interferences caused by non-target components in SXD, intestinal bacteria and incubation solution. In addition, because a large number of samples must be analyzed for pharmacokinetic studies, a rapid, simple and cost-effective sample preparation method is required.

Optimization of sample preparation. Sample preparation is a critical procedure for eliminating interference from the sample matrix and achieving satisfactory recovery. Based on a limited survey of the current literature, ${ }^{\mathbf{3 8 - 4 1}}$ both classical liquid-liquid extraction (LLE) and protein precipitation (PPT) are among the most widely utilized sample preparation techniques for the extraction of analyzed components from a cultured mixture. Reports on SALLE with water miscible organic solvents and inorganic salts as the salting-out agent for the preparation of biological samples are scarce, especially for intestinal bacteria.

To obtain the 16 analyzed components with minimal matrix interference, PPT (acetone and acetonitrile), LLE (ethyl acetate and water-saturated $n$-butanol), solid-phase extraction (SPE) (Oasis HLB and Agela Cleanert PEP-SPE) and SALLE (acetonitrile with $\mathrm{NaCl}$ as the salting-out reagent and acetonitrile/watersaturated $n$-butanol $(1: 1, \mathrm{v} / \mathrm{v})$ with $\mathrm{NaCl})$ were evaluated. PPT using acetone or acetonitrile and LLE using water-saturated $n$-butanol were not considered because the ion intensities of the analyzed components were reduced due to matrix effects, although the extraction recoveries were relatively high. LLE using ethyl acetate and SALLE using acetonitrile and $\mathrm{NaCl}$ provided low extraction recoveries. Although the recovery range of SPE was acceptable, it was time consuming and cost prohibitive. Moreover, matrix interference were not completely eliminated for all of the analyzed components when SPE was performed.
SALLE using acetonitrile/water-saturated $n$-butanol $(1: 1$, $\mathrm{v} / \mathrm{v}$ ) with $\mathrm{NaCl}$ could provide relatively high extraction recoveries, low matrix interferences and good repeatability for most of analyzed components satisfying the requirements of quantification for determination of pharmacokinetic parameters. Finally, the SALLE method was performed using acetonitrile/ water-saturated $n$-butanol $(1: 1, \mathrm{v} / \mathrm{v})$ and $\mathrm{NaCl}$ to prepare the samples.

However, quantification of glycyrrhetic acid, a metabolite of glycyrrhizic acid by intestinal bacteria, was compromised in this study due to its low recovery. As a saponin aglycone, the low polarity of glycyrrhetic acid makes its extraction difficult from bacterial incubation solution using acetonitrile/water-saturated $n$-butanol $(1: 1, \mathrm{v} / \mathrm{v})$ as the extract solvent. Fortunately, the recoveries of glycyrrhetic acid and other target components in further study can be improved by controlling the $\mathrm{pH}$ values and choosing appropriate solvents (acetone, methanol, ethanol and acetonitrile, etc.) for SALLE.

Compared with conventional LLE and PPT, SALLE in the present study provided a cleaner extract, effectively removing macromolecules. High extraction efficiencies were obtained for all 16 components of SXD via SALLE without the occurrence of emulsification. Compared with SPE, SALLE used much less solvent and was much faster and simpler to perform. Moreover, this was the first time that SALLE has been performed using acetonitrile/water-saturated $n$-butanol $(1: 1, \mathrm{v} / \mathrm{v})$ with $\mathrm{NaCl}$ for the extraction of target components in bacterial incubation solution.

Optimization of LC-MS/MS. Each standard solution of the analyzed components and the ISs $\left(5 \mu \mathrm{g} \mathrm{mL} \mathrm{m}^{-1}\right)$ in $50 \%$ methanol was directly infused into the mass spectrometer, which was operated in ESI mode using a syringe pump operating at a continuous flow rate of $7 \mu \mathrm{L} \mathrm{min}{ }^{-1}$. The response observed in the negative ionization mode was higher and more stable than that obtained in the positive mode for oroxylin A, baicalin, baicalein, wogonoside, wogonin, chrysin, scutellarin, glycyrrhizic acid, liquiritin and liquiritigenin. However, the positive 
Table 3 Intra-day, inter-day accuracy and precision for each analyte

\begin{tabular}{|c|c|c|c|c|c|}
\hline Components & Conc. (ng ml ${ }^{-1}$ ) & \multicolumn{2}{|l|}{ Intra-day $(n=5)$} & \multicolumn{2}{|l|}{ Inter-day $(n=5)$} \\
\hline & 20 & 8.60 & -5.02 & 4.77 & -8.95 \\
\hline & 200 & 3.30 & 0.58 & 4.49 & -4.26 \\
\hline & 400 & 3.27 & -2.38 & 6.15 & -7.46 \\
\hline & 200 & 4.09 & 7.00 & 6.30 & 0.07 \\
\hline & 800 & 7.45 & -5.05 & 5.33 & -9.25 \\
\hline \multirow[t]{4}{*}{ Baicalein } & 20 & 11.74 & 10.20 & 7.68 & 16.50 \\
\hline & 40 & 4.59 & 4.30 & 10.17 & -6.26 \\
\hline & 200 & 2.40 & 8.25 & 2.97 & 4.94 \\
\hline & 800 & 3.61 & -7.58 & 5.67 & -10.21 \\
\hline \multirow[t]{4}{*}{ Wogonin } & 20 & 8.00 & -14.98 & 3.16 & -17.43 \\
\hline & 40 & 7.85 & -12.43 & 2.56 & -12.62 \\
\hline & 200 & 2.26 & 12.00 & 5.11 & 6.70 \\
\hline & 800 & 4.17 & 1.13 & 10.08 & -4.69 \\
\hline \multirow[t]{4}{*}{ Chrysin } & 2 & 8.37 & -4.83 & 6.25 & -7.78 \\
\hline & 4 & 1.87 & -1.95 & 7.93 & -3.54 \\
\hline & 40 & 2.12 & -2.55 & 2.89 & 0.33 \\
\hline & 160 & 4.41 & -0.95 & 7.55 & -4.45 \\
\hline \multirow[t]{4}{*}{ Scutellarin } & 3 & 19.34 & -0.70 & 9.40 & 10.90 \\
\hline & 6 & 2.53 & 3.75 & 7.17 & 2.88 \\
\hline & 60 & 6.48 & -13.68 & 3.80 & -10.00 \\
\hline & 240 & 4.69 & -9.53 & 6.26 & -4.58 \\
\hline Glycyrrhizic acid & 12 & 4.24 & -11.95 & 3.75 & -9.55 \\
\hline \multirow{3}{*}{ Liquiritigenin } & 20 & 4.93 & 0.88 & 4.72 & -2.45 \\
\hline & 200 & 2.56 & 2.88 & 2.56 & 5.96 \\
\hline & 800 & 1.38 & 0.58 & 8.86 & 0.43 \\
\hline \multirow[t]{4}{*}{ Isoliquiritin } & 3 & 4.72 & 13.00 & 8.40 & 6.67 \\
\hline & 6 & 5.76 & -4.58 & 9.41 & -5.72 \\
\hline & 60 & 5.32 & -9.90 & 14.23 & -2.91 \\
\hline & 240 & 5.87 & -9.62 & 4.94 & -7.06 \\
\hline Isoliquiritigenin & 1 & 14.37 & 14.00 & 8.26 & 7.71 \\
\hline & 2 & 9.97 & -6.90 & 6.32 & -10.88 \\
\hline & 20 & 3.00 & -8.67 & 2.48 & -7.03 \\
\hline & 80 & 3.89 & 0.57 & 11.26 & -7.93 \\
\hline Berberine & 50 & 9.47 & -8.20 & 17.86 & 5.07 \\
\hline & 100 & 1.90 & 2.40 & 2.97 & 5.58 \\
\hline & 400 & 3.24 & 3.60 & 4.70 & 1.93 \\
\hline & 800 & 3.62 & -10.42 & 11.24 & -0.24 \\
\hline Coptisine & 25 & 9.87 & -10.58 & 16.68 & 1.38 \\
\hline & 50 & 7.45 & 0.60 & 2.46 & 1.51 \\
\hline & 200 & 3.88 & 3.00 & 1.34 & 1.76 \\
\hline & 400 & 4.41 & -9.86 & 5.86 & -4.41 \\
\hline Palmatine & 25 & 7.54 & 14.67 & 3.58 & 11.83 \\
\hline & 50 & 0.82 & 9.60 & 7.31 & 4.22 \\
\hline & 200 & 3.98 & -1.18 & 3.41 & -3.51 \\
\hline & 400 & 4.38 & -10.62 & 9.99 & -3.83 \\
\hline
\end{tabular}


Table 3 (Contd.)

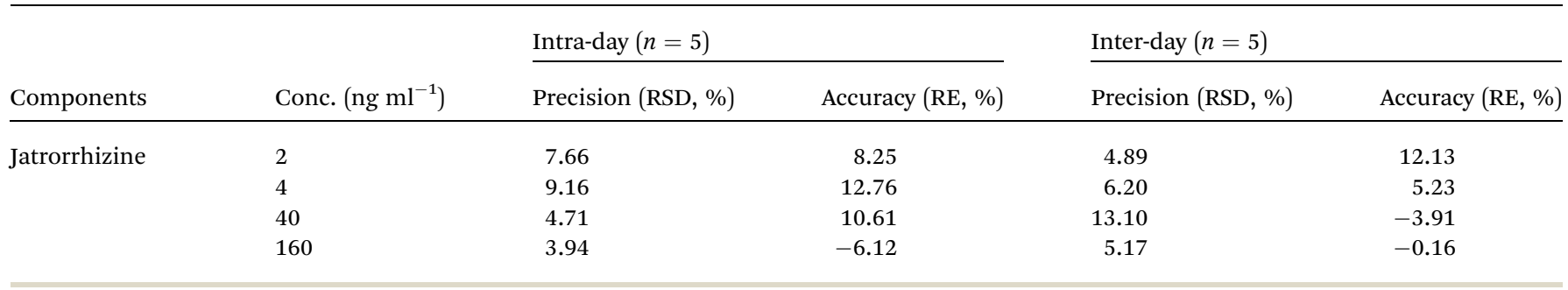

ionization mode was more sensitive and suitable for isoliquiritin, isoliquiritigenin, berberine, coptisine, palmatine and jatrorrhizine.

To achieve the maximum sensitivity and response, the precursor and product ion pairs for MRM detection, as well as their corresponding DP, CE, EP and CXP values, were optimized for the quantification of each analyzed component. The results are given in Table 1.

To improve resolution and decrease runtime, methanol, acetonitrile, ammonium acetate and formic acid were tested as potential mobile phases. Acetonitrile and $0.1 \%$ aqueous formic acid were employed as the mobile phase because the best separation of all the analyzed components from each other and the minimal influence of the matrix effect were achieved. Moreover, to improve the separation efficiency for the four alkaloids and isoliquiritin, the chromatographic condition II (in which the proportion of the organic phase between 12 and 16 min was decreased compared with that in the chromatographic condition I) was performed to obtain a better separation for berberine, coptisine, palmatine, jatrorrhizine and isoliquiritin.

\subsection{Method validation}

Selectivity. The chromatograms of the blank incubation solution of inactivated intestinal bacteria, the blank incubation solution of inactivated intestinal bacteria spiked with the standard solutions and three ISs in LLOQ, and the samples obtained after $2 \mathrm{~h}$ of incubation with intestinal bacteria are shown in Fig. 2A and B. Under the optimized LC-MS/MS conditions, no endogenous interference was observed in the incubation solution of intestinal bacteria.

Linearity and LLOQ. The calibration curves and LLOQs of all 16 analyzed components are summarized in Table 2. The correlation coefficient $(r)$ for each calibration curve was greater than 0.99 , which was indicative of good linearity in the linear range. For all of the analyzed components, the RSDs for the precision and the REs for the accuracy of the LLOQs for intraday and inter-day determinations were less than $19.34 \%$ and ranged between $-17.43 \%$ and $16.50 \%$, respectively. The quantification was sufficiently sensitive for the in vitro pharmacokinetic analysis of all 16 analyzed components in the incubation system of rat intestinal bacteria.

Precision and accuracy. As shown in Table 3, for all three concentrations (low, medium and high), the precision, measured as the RSD, was less than $15 \%$, and the accuracy, measured as the RE, was within $\pm 15 \%$. At the LLOQ, the RSD was less than $20 \%$ and the RE was within $\pm 20 \%$. These results indicated that the method was reliable and reproducible for the determination of all 16 analyzed components in rat intestinal bacterial incubation systems in vitro.

Extraction recovery and matrix effect. The extraction recoveries (Table 4) of the analyzed components at low, medium and high concentrations of the QC samples ranged from $54.50 \%$ to $94.71 \%$, with RSDs of less than $9.95 \%$. The wide range of extraction recoveries for the analyzed components was attributed to the variations in the polarities and physic-chemical characteristics among the analyzed components. Additionally, the extraction recoveries of naringin (IS1), naringenin (IS2) and tetrahydropalmatine (IS3) were $89.91 \pm 3.87 \%, 77.22 \pm 6.44 \%$ and $108.16 \pm 7.04 \%$, respectively. As shown in Table 5, the RSDs of the IS-normalized MF for the analyzed components from the six batches of matrix at low and high concentrations were less than $14.8 \%$, indicating that the matrix effect was negligible for this assay.

Dilution integrity. QC samples at concentrations two times higher than the upper limit of the calibration curve were diluted by a factor of ten with post-extracted blank intestinal bacterial incubation solution containing the ISs. The RSDs for the dilution integrity (Table 6) were less than $6.28 \%$ and the REs ranged from $-10.3 \%$ to $14.6 \%$. These data supported a 10 -fold sample dilution for analysis.

Stability. The short-term stability, long-term stability and freeze-thaw stability were evaluated using the mean concentrations of the QC samples at all three levels. The results listed in Table 4 indicated that all of the analyzed components were stable in the blank incubation solution of inactivated intestinal bacteria for three freeze-thaw cycles and for $48 \mathrm{~h}$ at $10^{\circ} \mathrm{C}$. The analyzed components were also demonstrated to be stable for two months at $-80{ }^{\circ} \mathrm{C}$.

\subsection{Incidence of CPT-11-induced diarrhea}

The animals' body weight (Fig. 3) and diarrheal symptoms were monitored after the first administration of CPT-11 throughout the experimental period. The body weight ratio in the GT group declined significantly $(p<0.05)$, reaching its lowest point at day 5 (Fig. 3). Delayed-onset diarrhea started at day 4 and became worst at day 5 , with an average diarrhea score of 2.1.

\subsection{Application of the method to pharmacokinetic study}

The developed ultra-high-performance liquid chromatographytandem mass spectrometry (UHPLC-MS/MS) method was 
Table 4 Extract recoveries and stabilities of 16 components

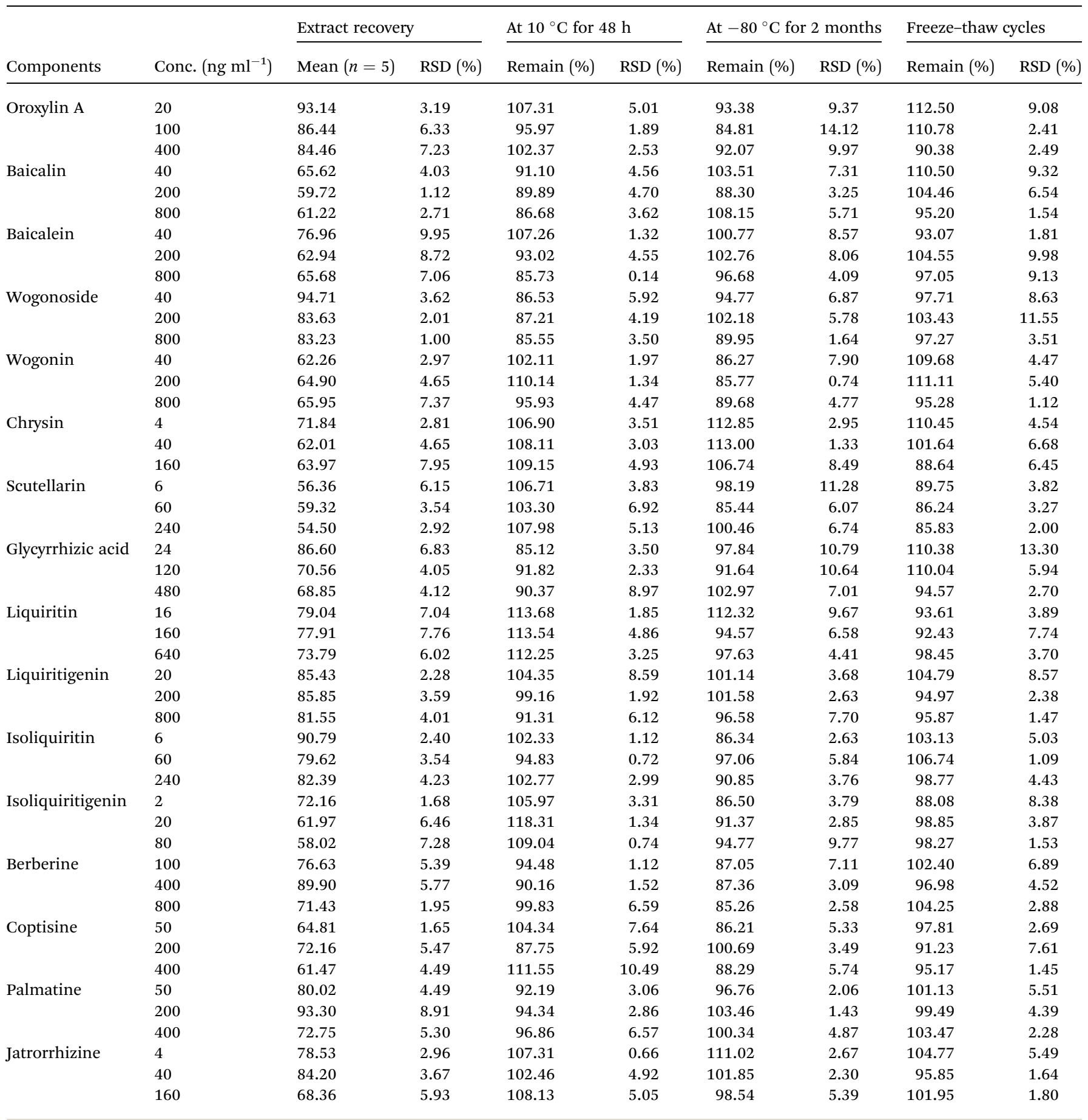

applied to determine the concentrations of all 16 analyzed components in the intestinal bacterial incubation system at 0 , $2,4,6,8,12,16,20,24,28,32,36$ and $48 \mathrm{~h}$ in groups $\mathrm{C}$ and GT. The mean concentration-time curves of the 16 analyzed components in the bacterial incubation solutions of normal rats (group C) and those with CPT-11-induced gastrointestinal toxicity (group GT) are shown in Fig. 4. A non-compartment model was used to calculate the pharmacokinetic parameters of the 16 analyzed components in the two groups, which are shown in Tables 7 and 8.
A negative control experiment was carried out to demonstrate that the changes in the concentrations of the 16 analyzed components of SXD in the intestinal bacterial incubation system were caused by the bacteria. The SXD extract was anaerobically incubated in GAM broth in the absence of the intestinal bacteria for $48 \mathrm{~h}$ at $37{ }^{\circ} \mathrm{C}$, then processed and analyzed via the proposed method. The peak areas obtained at $48 \mathrm{~h}$ were compared with those obtained at $0 \mathrm{~h}$. The observed deviations in the peak area were calculated as percentages. The results revealed that the peak area percentages of the analyzed 
Table 5 The matrix effects of 16 components

\begin{tabular}{|c|c|c|c|c|c|}
\hline Components & Conc. (ng ml ${ }^{-1}$ ) & $\begin{array}{l}\text { RSD of IS normalised } \\
\text { MF }(\%)\end{array}$ & Components & Conc. $\left(\mathrm{ng} \mathrm{m} \mathrm{m}^{-1}\right.$ ) & $\begin{array}{l}\text { RSD of IS normalised } \\
\text { MF (\%) }\end{array}$ \\
\hline Oroxylin A & 20 & 7.72 & Liquiritin & 16 & 12.9 \\
\hline \multirow[t]{2}{*}{ Baicalin } & 40 & 13.0 & Liquiritigenin & 20 & 6.96 \\
\hline & 800 & 13.5 & & 800 & 10.4 \\
\hline Baicalein & 40 & 10.4 & Isoliquiritin & 6 & 14.1 \\
\hline & 800 & 14.8 & & 80 & 6.42 \\
\hline \multirow[t]{2}{*}{ Wogonin } & 40 & 6.54 & Berberine & 100 & 8.53 \\
\hline & 800 & 13.8 & & 800 & 10.9 \\
\hline \multirow[t]{2}{*}{ Chrysin } & 4 & 10.6 & Coptisine & 50 & 10.5 \\
\hline & 160 & 11.8 & & 400 & 9.23 \\
\hline Scutellarin & 6 & 12.7 & Palmatine & 50 & 7.88 \\
\hline
\end{tabular}

components were within the range of $92.7 \%$ to $109.3 \%$, with RSDs of less than $3.45 \%(n=5)$, which implied that the concentrations of the analyzed components were not affected by the anaerobic medium broth or cultured conditions.

Metabolism of ten components by intestinal bacteria. Incubation of the SXD extract for $48 \mathrm{~h}$ with rat intestinal bacteria in vitro decreased the concentrations of baicalin, wogonoside, scutellarin, glycyrrhizic acid, liquiritin, isoliquiritin, berberine, coptisine, palmatine, and jatrorrhizine, which indicated that the intestinal bacteria were active to the degradations of these components in SXD.

Among the three flavonoid glycosides (scutellarin, baicalin and wogonoside) of SXD, the CL of scutellarin was greater than that of baicalin and wogonoside in the control group. The degree of metabolism was closely related to the chemical structure. Compared with the structures of baicalin and wogonoside, scutellarin exhibit one more $4^{\prime}$-position hydroxyl, which contributes to its excellent degree of microbial degradation. ${ }^{42}$ The lower CL of wogonoside indicated its stability to the bacteria, which resulted from the steric hindrance of methoxyl at the 8-position. In the GT group, the significantly increased CLs of baicalin, wogonoside and scutellarin implied that the bacteria from rats with CPT-11induced gastrointestinal toxicity catalyzed the degradations of three flavonoid glycosides. CPT-11 has been reported to increase the levels of Enterococcus spp., Clostridium spp., Escherichia coli,
Serratia spp., Staphylococcus spp., Peptostreptococcus spp. and Bacillus spp. in the colon. ${ }^{9,10}$ Among them, Clostridium spp., Escherichia coli and Staphylococcus spp. produced $\beta$-glucuronidase. ${ }^{10}$ The increased level of the above three species of bacteria up-regulated the expression of $\beta$-glucuronidase. The extent of the increase in the CL of wogonoside (approximately 4.6-fold) was greater than that of baicalin (approximately 2.4-fold) and scutellarin (approximately 2.2-fold), although the three flavonoid glycosides were all hydrolyzed by bacterial $\beta$-glucuronidase. The other metabolic pathway/degree of wogonoside was presumed to be altered in GT group. Moreover, the exposure levels $\left(\mathrm{AUC}_{0-t}\right)$ and the $\mathrm{MRT}_{0-t}$ of baicalin, wogonoside and scutellarin decreased in the GT group, which was indicative of an increase in their biotransformation rate.

In contrast to the above flavonoid glycosides, the concentration of liquiritin (a flavanone glycoside) in group $\mathrm{C}$ declined rapidly, reaching $10.2 \%$ of the initial level at $20 \mathrm{~h}$, while the concentration in group GT declined to $4.12 \%$ at $16 \mathrm{~h}$. The metabolic rate is related to the type and site of glycosidic linkage. Moreover, liquiritin undergoes deoxygenation and acetylation by bacterial enzymes besides hydrogenation, methylation and deglycosylation which are the main metabolic pathways of baicalin, wogonoside and scutellarin. ${ }^{43}$ The $\mathrm{MRT}_{0-t}$ of liquiritin decreased in the group GT, while the $\mathrm{AUC}_{0-t}$ of liquiritin was not significantly different from that in

Table 6 The dilution integrity for each analyte at dilution factor of ten

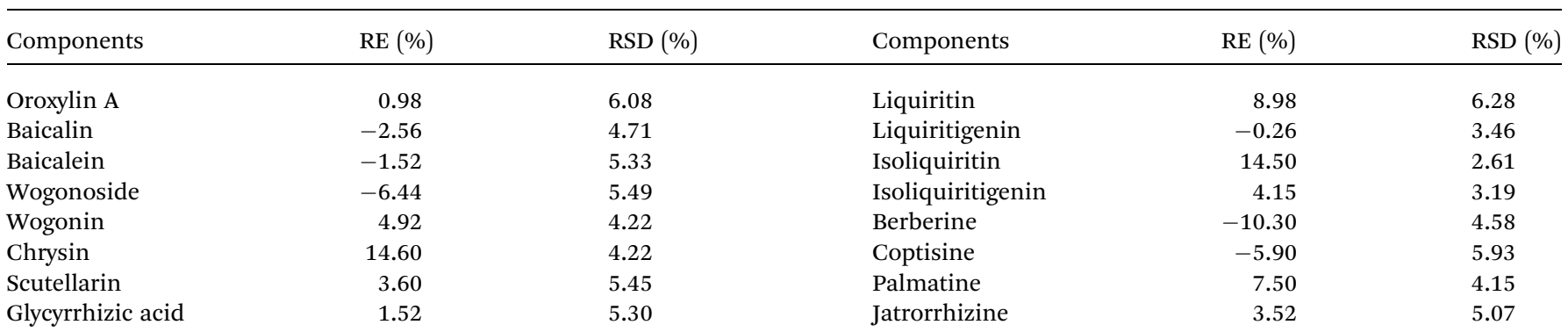




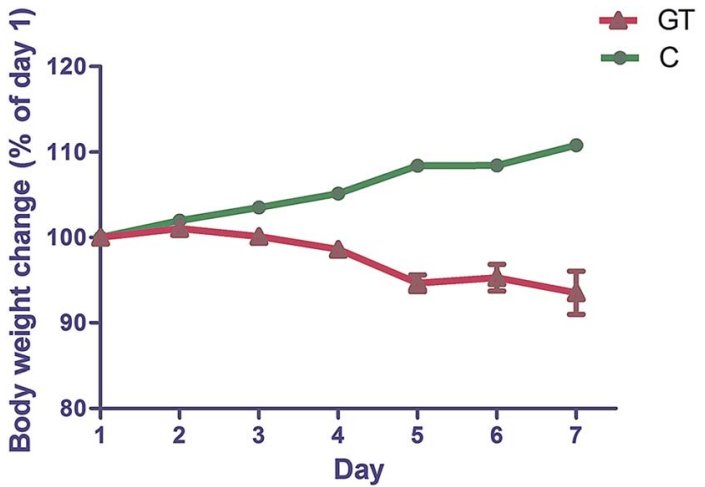

Fig. 3 Body weight changes of normal rats (C) and rats with CPT-11 induced gastrointestinal toxicity (GT).

group C. This observation indicated that liquiritin could be completely degraded by intestinal bacteria within $48 \mathrm{~h}$ in both groups. However, CPT-11 could influence the bacteriaassociated metabolic pathway or/and velocity of liquiritin. The increased level of Clostridium spp. by CPT-11 was speculated to accelerate the degradation rate of liquiritin. ${ }^{43}$ Similar changes were observed in the pharmacokinetic parameters of isoliquiritin, which exhibits a similar structure to that of liquiritin.

Although glycyrrhizic acid and baicalin are both glucuronide conjugates of aglycones, the CL of glycyrrhizic acid was significantly lower than that of the flavonoid glycosides. These results were supported by the report that the $\beta$-glucuronidase hydrolyzing glycyrrhizic acid might be different from the enzyme hydrolyzing baicalin, although the two compounds are both metabolized by $\beta$-glucuronidases. ${ }^{44}$ The former aimed to hydrolyze $\beta$-D-diglucuronide, while the latter might select $\beta$-Dmonoglucuronide to hydrolyze. Notably, there were no significantly differences in the pharmacokinetic parameters of glycyrrhizic acid between the two groups in the present study. It can be speculated that CPT- 11 alters the activity of the $\beta$ glucuronidase hydrolyzing $\beta$-D-monoglucuronide but not the enzyme hydrolyzing $\beta$-D-diglucuronide.

The concentration-time courses of the four alkaloids showed that the degradations of alkaloids by intestinal bacteria were relatively slow, and there were no significant differences in the pharmacokinetic parameters between the two groups. The slightly increased concentration of palmatine from 32 to $48 \mathrm{~h}$ resulted from the biotransformation of other alkaloids in Coptis chinensis. ${ }^{45}$ The increased concentration of berberine from 36 to
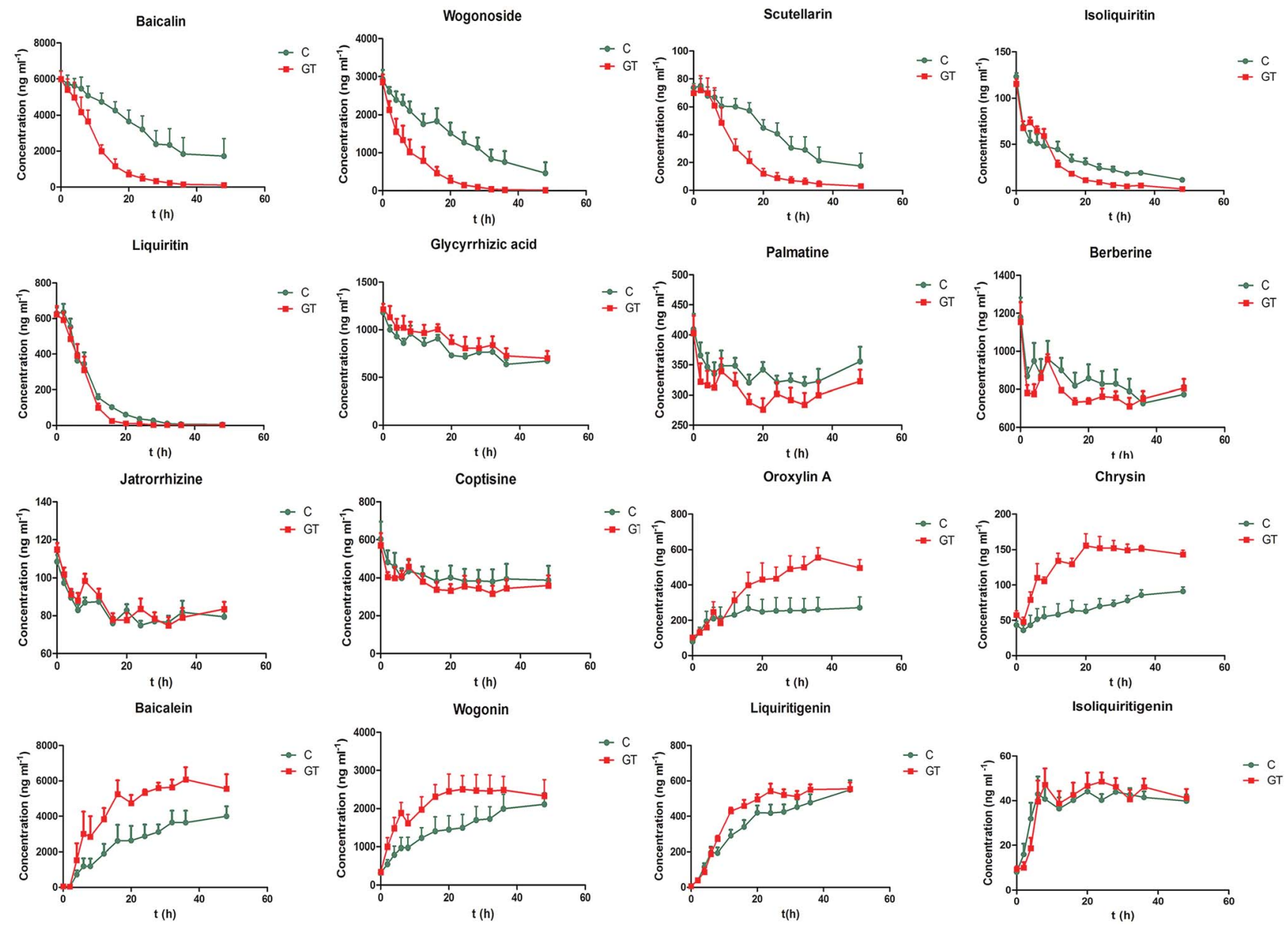

Fig. 4 Mean concentration-time profiles of 16 components. 
Table 7 The pharmacokinetic parameters of glycosides and alkaloids in the intestinal bacteria incubation solution of normal rats (group C) and those with CPT-11 induced gastrointestinal toxicity (group GT) (mean $\pm \mathrm{SD}, n=15)^{a}$

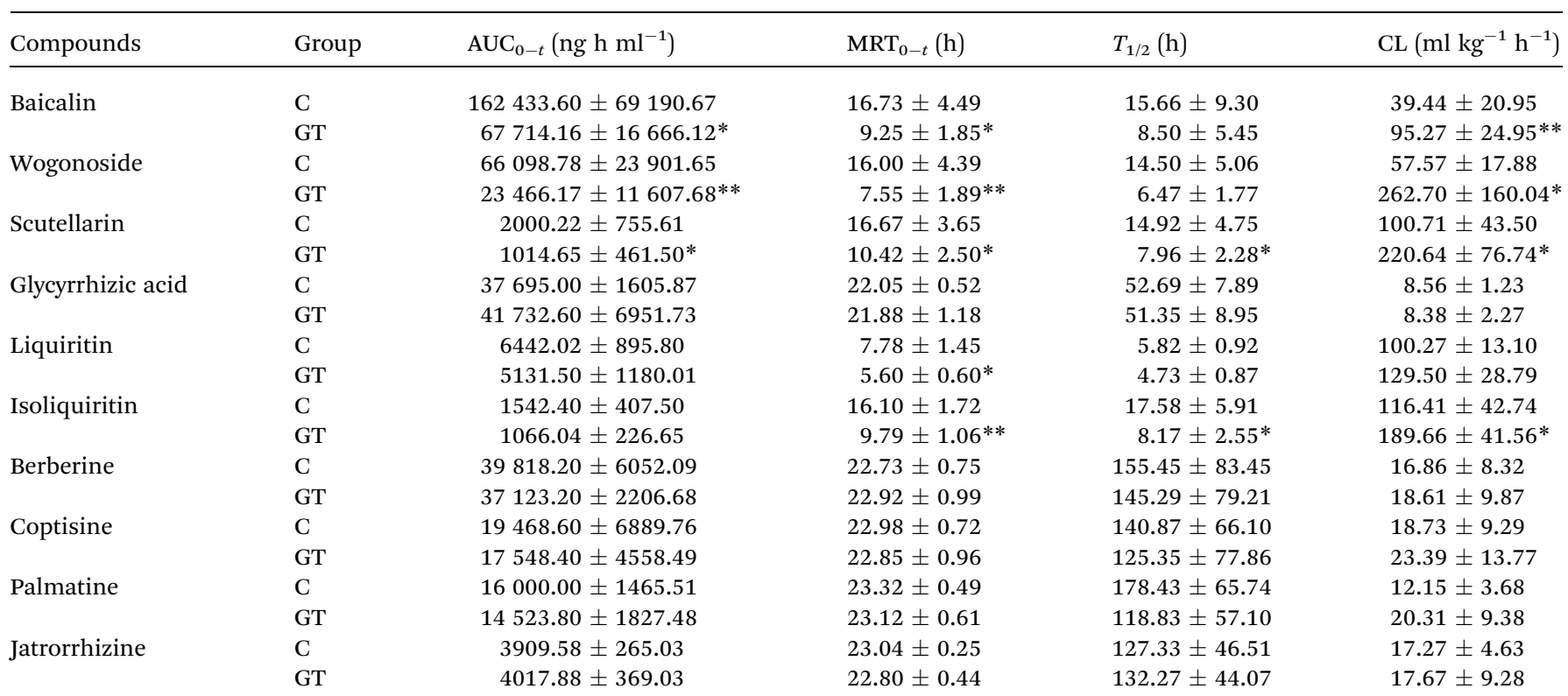

${ }^{a}$ Statistical difference between group C and GT, ${ }^{*} p<0.05,{ }^{* *} p<0.01$.

$48 \mathrm{~h}$ could be attributed to the oxidization of its metabolite dihydroberberine back to berberine. ${ }^{27}$

Production of six components by intestinal bacteria. Baicalein, wogonin, chrysin, oroxylin A, liquiritigenin, and isoliquiritigenin were produced during the incubation experiment, although the six components were identified in SXD previously. The larger $\mathrm{AUC}_{0-t}$ of baicalein and chrysin; the higher $C_{\max }$ of baicalein, chrysin and oroxylin A; and the shorter $T_{\max }$ of wogonin in group GT $(p<0.05, p<0.01)$ could be explained as a result of the increased biotransformation rates of the corresponding glycosides. In group GT, the decreased concentrations of the six aglycones at the later stage of incubation could be due to the degradation of aglycones by altered intestinal bacteria. $^{46}$
CPT-11 may induce changes in the metabolic behavior of glycosides and aglycones due to its impact on intestinal bacteria, which accelerates the degradation rate of glycosides to improve the production of aglycones. Increased accumulation of aglycones produced by intestinal bacteria in the intestine might mean improving absorption of aglycones and increasing bioavailability of aglycones. Flavonoid aglycones resulting from the metabolism of the corresponding glycoside by intestinal bacteria, such as baicalein, chrysin, oroxylin A and wogonin, have anti-inflammatory effects, ${ }^{31}$ which may alleviate CPT-11induced diarrhea. Moreover, chrysin has been shown to upregulate UGT1A1 to improve the conversion of $\mathrm{SN}-38$ to $\mathrm{SN}$ $38 \mathrm{G}$ in the gastrointestinal tract. ${ }^{47}$ Although CPT-11 does not alter the bacteria-associated metabolic behavior of berberine,

Table 8 The pharmacokinetic parameters of aglycones in the intestinal bacteria incubation solution of normal rats (group C) and those with CPT-11 induced gastrointestinal toxicity (group GT) (mean $\pm \mathrm{SD}, n=15)^{a}$

\begin{tabular}{|c|c|c|c|c|}
\hline Compounds & Group & $\mathrm{AUC}_{0-t}\left(\mathrm{ng} \mathrm{h} \mathrm{ml} \mathbf{H}^{-1}\right)$ & $C_{\max }\left(\mathrm{ng} \mathrm{ml}^{-1}\right)$ & $T_{\max }(\mathrm{h})$ \\
\hline \multirow[t]{2}{*}{ Baicalein } & $\mathrm{C}$ & $127761.98 \pm 50920.84$ & $4082.50 \pm 1123.58$ & $36.00 \pm 8.64$ \\
\hline & GT & $221389.61 \pm 37170.01 *$ & $6825.00 \pm 1118.23^{*}$ & $32.00 \pm 13.47$ \\
\hline \multirow[t]{2}{*}{ Wogonin } & $\mathrm{C}$ & $72504.30 \pm 32315.43$ & $2189.00 \pm 678.59$ & $43.20 \pm 6.57$ \\
\hline & GT & $104497.00 \pm 31749.46$ & $2714.00 \pm 873.32$ & $21.60 \pm 17.52^{*}$ \\
\hline \multirow[t]{2}{*}{ Chrysin } & $\mathrm{C}$ & $3324.62 \pm 900.04$ & $98.66 \pm 17.32$ & $39.20 \pm 13.97$ \\
\hline & GT & $6411.08 \pm 530.95 * *$ & $174.00 \pm 25.93^{* *}$ & $28.40 \pm 15.52$ \\
\hline \multirow[t]{2}{*}{ Oroxylin A } & $\mathrm{C}$ & $11539.89 \pm 6800.95$ & $301.20 \pm 149.08$ & $34.40 \pm 14.31$ \\
\hline & GT & $19463.42 \pm 4701.65$ & $591.80 \pm 129.03^{*}$ & $30.40 \pm 6.69$ \\
\hline \multirow[t]{2}{*}{ Liquiritigenin } & $\mathrm{C}$ & $17792.71 \pm 3615.60$ & $550.60 \pm 119.37$ & $42.40 \pm 12.52$ \\
\hline & GT & $21028.03 \pm 2263.81$ & $577.60 \pm 68.70$ & $32.00 \pm 10.20$ \\
\hline \multirow[t]{2}{*}{ Isoliquiritigenin } & $\mathrm{C}$ & $1872.57 \pm 271.96$ & $51.34 \pm 10.80$ & $26.80 \pm 15.47$ \\
\hline & GT & $1957.04 \pm 463.40$ & $53.16 \pm 11.26$ & $23.20 \pm 16.59$ \\
\hline
\end{tabular}

${ }^{a}$ Statistical difference between group C and GT, ${ }^{*} p<0.05,{ }^{* *} p<0.01$. 
berberine could be transformed to its intestine-absorbable form by the intestinal bacteria and enter into the blood to exert its anti-diarrheal action. ${ }^{27,48}$ Therefore, the interactions between CPT-11, intestinal bacteria and SXD are proposed, in which CPT-11 alters the intestinal bacteria qualitatively and quantitatively and thus changes metabolic behavior of SXD, resulting in protective constituents from SXD alleviating the gastrointestinal toxicity induced by CPT-11 in turn.

\section{Conclusion}

In the present study, a salting-out sample preparation and UHPLC-MS/MS method was developed for the determination of oroxylin A, baicalin, baicalein, wogonoside, wogonin, chrysin, scutellarin, glycyrrhizic acid, liquiritin, liquiritigenin, isoliquiritin, isoliquiritigenin, berberine, coptisine, palmatine and jatrorrhizine in a complex incubation system for rat intestinal bacteria for the first time. The method was rapid, simple and efficient, and help solving critical problems for analyses of target components in complex biological samples, such as eliminating interferences caused by biological matrix and nontarget components, especially for analyses of those in a complex mixture consisting of TCM and intestinal bacteria incubation system. The proposed method was successfully applied to the intestinal bacteria-associated pharmacokinetics of the abovementioned components in vitro, offering technical references in the field of research on the interaction between intestinal bacteria and TCM. This study was also the first to compare the pharmacokinetic parameters of the 16 components in bacterial incubation solutions from normal rats and those with CPT-11induced gastrointestinal toxicity. Our findings will be useful for achieving a deeper understanding of the mechanisms involved in the changes in intestinal bacteria induced by CPT-11 and further pharmacokinetic comparisons of the components between normal rats and those with CPT-11-induced gastrointestinal toxicity in vivo. In summary, the developed UHPLC-MS/ MS method is useful for the evaluation of SXD components in biological processes, and the intestinal bacteria-based pharmacokinetic method applied in the present investigation will likely be beneficial to the study of interactions between TCMs and chemical drugs in current clinical practice.

\section{Conflicts of interest}

There are no conflicts of interest to declare.

\section{Acknowledgements}

This work was financially supported by the National Natural Science Foundation of China (grant no. 81274054), the Beijing Municipal Natural Science Foundation (grant no. 7142109), the Science and Technology Major Programmer for Major Drug Discovery (grant no. 2012ZX09301002-001-028) and the CAMS Innovation Fund for Medical Sciences (CIFMS) (grant no. 2016I2M-1-012). The authors are very thankful to the Drug Discovery Facility, which belongs to the Center of Biomedical Analysis in Tsinghua University.

\section{References}

1 P. Herviou, D. Richard, L. Roche, J. Pinguet, F. Libert, A. Eschalier, X. Durando and N. Authier, J. Pharm. Biomed. Anal., 2016, 118, 284-291.

2 K. Takasuna, T. Hagiwara, M. Hirohashi, M. Kato, M. Nomura, E. Nagai, T. Yokoi and T. Kamataki, Cancer Chemother. Pharmacol., 1998, 42, 280-286.

3 A. Kurita, S. Kado, N. Kaneda, M. Onoue, S. Hashimoto and T. Yokokura, Cancer Chemother. Pharmacol., 2000, 46, 211220.

4 A. Kurita, S. Kado, N. Kaneda, M. Onoue, S. Hashimoto and T. Yokokura, Cancer Chemother. Pharmacol., 2003, 52, 349-360.

5 M. Onoue, A. Kurita, S. Kado, T. Matsumoto, N. Kaneda, K. Uchida, I. Kato and T. Yokokura, Cancer Chemother. Pharmacol., 2008, 61, 595-605.

6 K. Takasuna, T. Hagiwara, K. Watanabe, S. Onose, S. Yoshida, E. Kumazawa, E. Nagai and T. Kamataki, Cancer Chemother. Pharmacol., 2006, 58, 494-503.

7 A. Kurita, S. Kado, T. Matsumoto, N. Asakawa, N. Kaneda, I. Kato, K. Uchida, M. Onoue and T. Yokokura, Cancer Chemother. Pharmacol., 2011, 67, 201-213.

8 K. Takasuna, T. Hagiwara, M. Hirohashi, M. Kato, M. Nomura, E. Nagai, T. Yokoi and T. Kamataki, Cancer Res., 1996, 56, 3752-3757.

9 A. M. Stringer, R. J. Gibson, R. M. Logan, J. M. Bowen, A. S. J. Yeoh and D. M. K. Keefe, Cancer Biol. Ther., 2008, 7, 1919-1925.

10 A. M. Stringer, R. J. Gibson, R. M. Logan, J. M. Bowen, A. S. J. Yeoh, J. Burns and D. M. K. Keefe, Exp. Biol. Med., 2007, 232, 96-106.

11 T. Nakao, N. Kurita, M. Komatsu, K. Yoshikawa, T. Iwata, T. Utusnomiya and M. Shimada, J. Surg. Res., 2012, 173, 341-347.

12 A. Kato, J. Ueyama, F. Abe, K. Hotta, I. Tsukiyama, T. Oshima, F. Kondo, H. Saito and T. Hasegawa, Anticancer Res., 2011, 31, 2915-2922.

13 B. D. Wallace, H. Wang, K. T. Lane, J. E. Scott, J. Orans, J. S. Koo, M. Venkatesh, C. Jobin, L. A. Yeh, S. Mani and M. R. Redinbo, Science, 2010, 330, 831-835.

14 Y. Q. Gao, Y. C. Si, X. N. Liu, Q. F. Luo and X. Niu, Beijing Zhongyiyao Daxue Xuebao, 2004, 27, 47-49.

15 G. Chen, Y. Yang, M. Liu, Z. Teng, J. Ye, Y. Xu, X. Cai, X. Cheng, J. Yang, C. Hu, M. Wang and P. Cao, J. Ethnopharmacol., 2015, 166, 149-156.

16 L. Q. Jia and Y. N. Lou, The effect of Chinese herbs in prevention and treatment of side-effect associated with new anticancer agents, The 12th annual meeting of China association for science and technology: The function and status of traditional Chinese medicine in common health accident, Fujian, Nov 2010, pp. 113-116.

17 J. Wang, L. Q. Jia, H. Y. Tan, L. Pan, L. L. Yu and B. Deng, Chin. J. Integr. Tradit. West. Med., 2015, 35, 1236-1243.

18 C. Deng, B. Deng, L. Jia, H. Tan, P. Zhang, S. Liu, Y. Zhang, A. Song and L. Pan, J. Evidence-Based Complementary Altern. Med., 2017, DOI: 10.1155/2017/7350251. 
19 K. Takasuna, Y. Kasai, Y. Kitano, K. Mori, R. Kobayashi, T. Hagiware, K. Kakihata, M. Hirohashi, M. Nomura, E. Nagai and T. Kamataki, Jpn. J. Cancer Res., 1995, 86, 978-984.

20 T. Itoh, S. Itagaki, Y. Sumi, T. Hirano, I. Takemoto and K. Iseki, Cancer Chemother. Pharmacol., 2005, 55, 420-424.

21 P. Huang, S. Tan, Y. X. Zhang, J. S. Li, C. Chai, J. J. Li and B. C. Cai, J. Ethnopharmacol., 2014, 155, 649-664.

22 L. Wang, S. Y. Zhang, L. Chen, X. J. Huang, Q. W. Zhang, R. W. Jiang, F. Yao and W. C. Ye, Phytochem. Lett., 2014, 7, 89-92.

23 R. Krausse, J. Bielenberg, W. Blaschek and U. Ullmann, J. Antimicrob. Chemother., 2004, 54, 243-246.

24 T. Akao, Biol. Pharm. Bull., 2000, 23, 1418-1423.

25 D. H. Kim, I. S. Jang, H. K. Lee, E. A. Jung and K. Y. Lee, Arch. Pharmacal Res., 1996, 19, 292-296.

26 Q. C. Yang, W. H. Wu, F. M. Han and Y. Chen, J. Pharm. Pharmacol., 2009, 61, 647-652.

27 R. Feng, J. W. Shou, Z. X. Zhao, C. Y. He, C. Ma, M. Huang, J. Fu, X. S. Tan, X. Y. Li, B. Y. Wen, X. Chen, X. Y. Yang, G. Ren, Y. Lin, Y. Chen, X. F. You, Y. Wang and J. D. Jiang, Sci. Rep., 2015, 5, 12155.

28 S. Xing, M. Wang, Y. Peng, D. Chen and X. Li, J. Ethnopharmacol., 2014, 152, 183-189.

29 G. Peng, H. Guan, X. Wang and Y. Shi, Acta Pharm. Sin. B, 2017, 7, 193-201.

30 Y. Wang, R. Xu, J. Xiao, J. Zhang, X. Wang, R. An and Y. Ma, J. Pharm. Biomed. Anal., 2014, 88, 525-535.

31 L. Tong, M. Wan, L. Zhang, Y. Zhu, H. Sun and K. Bi, J. Pharm. Biomed. Anal., 2012, 70, 6-12.

32 H. J. Chung, S. Lim, I. S. Kim, Y. Bu, H. Kim, D. H. Kim and H. H. Yoo, Bull. Korean Chem. Soc., 2012, 33, 177-182.

33 H. L. Li, W. D. Zhang, C. Zhang, R. H. Liu, X. W. Wang, X. L. Wang, J. B. Zhu and C. L. Chen, J. Pharm. Biomed. Anal., 2006, 41, 1342-1346.
34 L. Liu and Z. Chen, Anal. Chim. Acta, 2012, 737, 99-104.

35 Z. J. Lin, S. X. Qiu, A. Wufuer and L. Shum, J. Chromatogr. B: Anal. Technol. Biomed. Life Sci., 2005, 814, 201-207.

36 Q. Zou, P. Wei, J. Li, Z. X. Ge and P. Ouyang, Biomed. Chromatogr., 2009, 23, 54-62.

37 European Medicines Agency, Committee for Medicinal Products for Human Use (CHMP), Guideline on Bioanalytical Method Validation, July 2011.

38 J. Xu, M. Zhao, D. Qian, E. X. Shang, S. Jiang, J. Guo, J. A. Duan and L. Du, J. Ethnopharmacol., 2014, 153, 368-374.

39 R. Xu, Y. Peng, M. Wang, L. Fan and X. Li, J. Ethnopharmacol., 2014, 158, 338-344.

40 D. Tang, Y. Yu, X. Zheng, J. Wu, Y. Li, X. Wu, Q. Du and X. Yin, J. Pharm. Biomed. Anal., 2014, 100, 1-10.

41 L. Yan, X. Yang, Z. Meng, Y. Yuan, W. Xiao, Z. Wang, W. Huang, Z. Yang and C. Zhang, J. Chromatogr. B: Anal. Technol. Biomed. Life Sci., 2014, 971, 81-88.

42 J. Xu, D. Qian, S. Jiang, J. Guo, E. X. Shang, J. A. Duan and J. Yang, Chromatographia, 2013, 76, 975-983.

43 W. Zhang, S. Jiang, D. Qian, E. Shang and J. Duan, Biomed. Chromatogr., 2014, 28, 1271-1277.

44 Y. S. Kim, J. J. Kim, K. H. Cho, W. S. Jung, S. K. Moon, E. K. Park and D. H. Kim, J. Microbiol. Biotechnol., 2008, 18, 1109-1114.

45 Y. Zhang, W. Wu, F. Han and Y. Chen, Biomed. Chromatogr., 2008, 22, 1360-1367.

46 Y. Pan, Z. Zhang, D. Ding and X. Jia, China J. Chin. Mater. Med., 2013, 38, 3239-3245.

47 T. Walle, Y. Otake, A. Galijatovic, J. K. Ritter and U. K. Walle, Drug Metab. Dispos., 2000, 28, 1077-1082.

48 C. Chen, Z. Yu, Y. Li, J. Fichna and M. Storr, Am. J. Chin. Med., 2014, 42, 1053-1070. 